$<$ Back to results 1 of 1

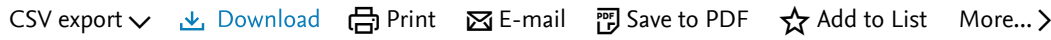

Full Text |View at Publisher|

Document type

Article

Source type

Journal

ISSN

09596526

DOI

10.1016/j.jclepro.2021.128042

View more $v$

Journal of Cleaner Production • Volume 315 • 15 September 2021 • Article number 128042

\section{Magnitudes of households' carbon footprint in Iskandar Malaysia: Policy implications for sustainable development}

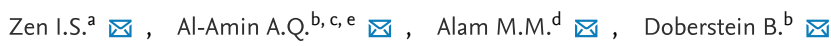

䀳 Save all to author list

${ }^{a}$ Dept. of Urban Regional Planning (URP), Kulliyyah of Architecture and Environmental Design, International Islamic University Malaysia (IIUM), Malaysia

${ }^{\mathrm{b}}$ Department of Geography and Environmental Management, University of Waterloo, ON, Canada

${ }^{c}$ Department of Urban Studies \& Planning, Massachusetts Institute of Technology, Cambridge, MA, United States

${ }^{d}$ Economic and Financial Policy Institute, School of Economics, Finance \& Banking (SEFB), Universiti Utara Malaysia (UUM), Malaysia View additional affiliations $\checkmark$

\section{Abstract}

Author keywords

Indexed keywords

SciVal Topics

Metrics

Funding details

Abstract

The carbon footprint of households is a significant contribution to global greenhouse gas emissions, accounting for $24 \%$ of total emissions. As a result, it is critical to quantify a household 's carbon footprint in order to reduce it over time. One of the best ways to measure carbon emitted from various sectors of the economy, including household daily activities, is to calculate a country's carbon footprint (CF). This study statistically examined the magnitude of households ' carbon footprints and their relationships with household daily activities and certain socio-economic demographic variables in Malaysia. Results revealed that the average household carbon footprint amounted to $11.76 \mathrm{t}-\mathrm{CO}_{2}$. The average also showed that the primary carbon footprint , $7.02 \mathrm{t}-\mathrm{CO}_{2}$ or $59.69 \%$ was higher compared to the secondary carbon footprint which was $4.73 \mathrm{t}-\mathrm{CO}_{2}$ or $40.22 \%$ and assessment revealed significant differences among household types. The largest carbon footprint was evident in a medium-high cost urban area, estimated at $20.14 \mathrm{t}-\mathrm{CO}_{2}$, while the carbon footprint found in a rural area was $9.58 \mathrm{t}-\mathrm{CO}_{2}$. In the latter, the primary carbon footprint was almost double the figure of $5.84 \mathrm{t}-\mathrm{CO}_{2}(61 \%)$ than the secondary carbon footprint of $3.73 \mathrm{t}-\mathrm{CO}_{2}(39 \%)$. The study reveals a higher carbon footprint in urban areas compared to rural ones depicting the effects of urbanisation and urban sprawl on household lifestyles and carbon footprints. Despite some limitations, the findings of this study will help policymakers design and implement stronger policies that enforce low- carbon activities and energy-saving goods and services in order to reduce urban Malaysia 's carbon footprint dramatically. (C) 2021 Elsevier Ltd
Cited by 0 documents

Inform me when this document is cited in Scopus:

Set citation alert >

Related documents

Estimation and determinants of the Philippines' household carbon footprint

Seriño, M.N.V., Klasen, S.

(2015) Developing Economies

Research on the carbon emissions of Beijing residents based on the inputoutput model

Zhang, Y. , Lei, Y.

(2017) Polish Journal of Environmental Studies

Income inequality and carbon dioxide emissions: The case of Chinese urban households

Golley, J., Meng, X.

(2012) Energy Economics

View all related documents based on references

Find more related documents in Scopus based on:

Authors > Keywords > 
Author keywords

Carbon emissions; Carbon footprint; Energy; Households; Lifestyle

Indexed keywords

SciVal Topics (i)

Metrics

Funding details

References (68)

View in search results format $>$

All

CSV export $\vee$ 合Print 四E-mail 退 Save to PDF Create bibliography

1 Chik, N.A., Rahim, K.A., Radam, A., Shamsudin, M.N.

$\mathrm{CO}_{2}$ emissions induced by households lifestyle in Malaysia

(2013) International Journal of Business and Society, 14 (3), pp. 344-357. Cited 6 times.

http://www.feb.unimas.my/index.php?

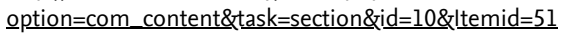

$2 \mathrm{ADB}$

Low-Carbon Green Growth in Asia: Policies and Practices

(2013). Cited 39 times.

Asian Development Bank Institute Japan

3 Akil, A.M., Ho, C.S.

Towards sustainable solid waste management: Investigating household participation in solid waste management (Open Access)

(2014) IOP Conference Series: Earth and Environmental Science, 18 (1), art. no.

012163. Cited 7 times.

http://www.iop.org/EJ/volume/1755-1315

doi: $10.1088 / 1755-1315 / 18 / 1 / 012163$

View at Publisher

4 Alfredsson, $\mathrm{E}$.

Green Consumption Energy Use and Carbon Dioxide Emission

(2002). Cited 18 times.

Umeå University Retrieved from

http://www.diva-portal.org/smash/get/diva2:144590/FULLTEXT01.pdf

5 Allison, E.H., Perry, A.L., Badjeck, M.-C., Neil Adger, W., Brown, K., Conway, D., Halls, A.S., (...), Dulvy, N.K.

Vulnerability of national economies to the impacts of climate change on fisheries (Open Access)

(2009) Fish and Fisheries, 10 (2), pp. 173-196. Cited 687 times.

doi: $10.1111 / j .1467-2979.2008 .00310 . x$

View at Publisher

6 Baiocchi, G., Minx, J.C., Hubacek, K.

The Impact of Social Factors and Consumer Behavior on CO2 Emissions in the UK: A

Panel Regression Based on Input-Output and Geo-Demographic Consumer Segmentation

Data

(2010)

Retrieved from

https://opus4.kobv.de/opus4-hsog/frontdoor/index/index/docld/1886 
$\square 7$ Bajželj, B., Richards, K.S., Allwood, J.M., Smith, P., Dennis, J.S., Curmi, E., Gilligan, C.A. Importance of food-demand management for climate mitigation (Open Access)

(2014) Nature Climate Change, 4 (10), pp. 924-929. Cited 361 times. http://www.nature.com/nclimate/index.html doi: $10.1038 /$ nclimate2353

View at Publisher

8 Barau, A.S.

Tension in the periphery: An analysis of spatial, public and corporate views on landscape change in Iskandar Malaysia

(2017) Landscape and Urban Planning, 165, pp. 256-266. Cited 10 times. www.elsevier.com/inca/publications/store/5/0/3/3/4/7 doi: 10.1016/j.landurbplan.2016.04.007

View at Publisher

9 Bin, S., Dowlatabadi, H.

Consumer lifestyle approach: a Paradigm for understanding the role of consumers in energy US and environmental impacts

(2002)

Doctoral Dissertation Carnegie Mellon University USA

10 Bin, S., Dowlatabadi, $\mathrm{H}$.

Consumer lifestyle approach to US energy use and the related $\mathrm{CO}_{2}$ emissions

(2005) Energy Policy, 33 (2), pp. 197-208. Cited 412 times.

http://www.journals.elsevier.com/energy=policy/

doi: 10.1016/S0301-4215(03)00210-6

View at Publisher

11 Borrion, A., Matsushita, J., Austen, K., Johnson, C., Bell, S.

Development of LCA Calculator to support community infrastructure codesign (Open Access)

(2019) International Journal of Life Cycle Assessment, 24 (7), pp. 1209-1221. Cited 4 times. http://www.springerlink.com/content/0948-3349 doi: 10.1007/s11367-018-1492-2

View at Publisher

12 Christis, M., Breemersch, K., Vercalsteren, A., Dils, E.

A detailed household carbon footprint analysis using expenditure accounts - Case of Flanders (Belgium)

(2019) Journal of Cleaner Production, 228, pp. 1167-1175. Cited 9 times.

https://www.journals.elsevier.com/journal-of-cleaner-production

doi: $10.1016 /$ j.jclepro.2019.04.160

View at Publisher

13 Cohen, L., Manion, L., Morrison, K.

Research Methods in Education

(2007). Cited 10872 times.

sixth ed. Routledge Falmer New York

14 Crenna, E., Secchi, M., Benini, L., Sala, S.

Global environmental impacts: data sources and methodological choices for calculating normalization factors for LCA (Open Access)

(2019) International Journal of Life Cycle Assessment, 24 (10), pp. 1851-1877. Cited 22

times.

http://www.springerlink.com/content/0948-3349

doi: 10.1007/s11367-019-01604-y

View at Publisher 
15 Climate Change Communication: Tomorrow's Climate, Today's Challenge (2007). Cited 2 times.

DEFRA, UK Department for Environment London Food and Rural Affairs

16 Druckman, A., Jackson, T.

The carbon footprint of UK households 1990-2004: A socio-economically disaggregated, quasi-multi-regional input-output model

(2009) Ecological Economics, 68 (7), pp. 2066-2077. Cited 378 times. doi: 10.1016/j.ecolecon.2009.01.013

View at Publisher

17 Farabi, A., Abdullah, A., Setianto, R.H.

Energy consumption, carbon emissions and economic growth in Indonesia and Malaysia (Open Access)

(2019) International Journal of Energy Economics and Policy, 9 (3), pp. 338-345. Cited 13

times.

http://www.econjournals.com/index.php/ijeep/article/download/6573/4345

doi: 10.32479/ijeep.6573

View at Publisher

18 Feng, Z.H., Zou, L.L., Wei, Y.M.

The impact of residents' lifestyle on $\mathrm{CO} 2$ emissions in China

(2010) Energy J. China, 32, pp. 37-40. Cited 9 times.

19 Fong, W.K.

Lessons Learned from Low-Carbon City Planning in Malaysia

(2013)

World Resources Institute April 29

http://www.wri.org/blog/2013/04/lessons-learned-low-carbon-city.planning-malaysia

20 Grunewald, N., Harteisen, M., Lay, J., Minx, J., Renner, S.

The carbon footprint of Indian Households

(2012). Cited 8 times.

General Conference of The International Association for Research in Income and Wealth

Boston. Retrieved from

http://www.iariw.org/papers/2012/GrunewaldPaper.pdf

21 Guerin, D.A., Yust, B.L., Coopet, J.G.

Occupant predictors of household energy behavior and consumption change as found in energy studies since 1975

(2000) Family and Consumer Sciences Research Journal, 29 (1), pp. 48-80. Cited 50 times. doi: 10.1177/1077727X00291003

View at Publisher

22 Gujba, H., Thorne, S., Mulugetta, Y., Rai, K., Sokona, Y. Financing Low Carbon

(2012)

23 Heinonen, J., Junnila, S.

A carbon consumption comparison of rural and urban lifestyles (Open Access)

(2011) Sustainability, 3 (8), pp. 1234-1249. Cited 52 times.

http://www.mdpi.com/2071-1050/3/8/1234/pdf

doi: $10.3390 /$ su3081234

View at Publisher 
24 Isaksen, E.T., Narbel, P.A.

A carbon footprint proportional to expenditure - A case for Norway? (Open Access)

(2017) Ecological Economics, 131, pp. 152-165. Cited 23 times. www.elsevier.com/inca/publications/store/5/0/3/3/0/5

doi: 10.1016/j.ecolecon.2016.08.027

View at Publisher

25 Rejuvenating Potential, Continuous Growth: Annual Report 2018

(2018)

Iskandar Malaysia Development Authority Malaysia

26 Jia, J., Fan, Y., Guo, X.

The low carbon development (LCD) levels' evaluation of the world's 47 countries (areas) by combining the FAHP with the TOPSIS method

(2012) Expert Systems with Applications, 39 (7), pp. 6628-6640. Cited 21 times. doi: 10.1016/j.eswa.2011.12.039

View at Publisher

27 Jones, C.M., Kammen, D.M.

Quantifying carbon footprint reduction opportunities for U.S. households and communities

(2011) Environmental Science and Technology, 45 (9), pp. 4088-4095. Cited 243 times. doi: $10.1021 /$ es102221h

View at Publisher

28 Kenny, T., Gray, N.F.

A preliminary survey of household and personal carbon dioxide emissions in Ireland (Open Access)

(2009) Environment International, 35 (2), pp. 259-272. Cited 44 times.

www.elsevier.com/locate/envint

doi: 10.1016/j.envint.2008.06.008

View at Publisher

29 Kerkhof, A.C., Benders, R.M.J., Moll, H.C.

Determinants of variation in household $\mathrm{CO}_{2}$ emissions between and within countries

(2009) Energy Policy, 37 (4), pp. 1509-1517. Cited 72 times.

doi: 10.1016/j.enpol.2008.12.013

View at Publisher

30 Koide, R., Lettenmeier, M., Kojima, S., Toivio, V., Amellina, A., Akenji, L.

Carbon footprints and consumer lifestyles: An analysis of lifestyle factors and gap analysis by consumer segment in Japan (Open Access)

(2019) Sustainability (Switzerland), 11 (21), art. no. 5983. Cited 13 times. https://res.mdpi.com/d_attachment/sustainability/sustainability-1l05983/article_deploy/sustainability-11-05983-v3.pdf doi: $10.3390 /$ sul1215983

View at Publisher

31 Krejcie, R.V., Morgan, D.W.

Determining sample size for research activities

(1970) Educ. Psychol. Meas., 30, pp. 607-610. Cited 4250 times.

Retrieved from

http://journals.sagepub.com/doi/pdf/10.1177/001316447003000308 
32 Larsen, H.N., Hertwich, E.G.

Identifying important characteristics of municipal carbon footprints

(2010) Ecological Economics, 70 (1), pp. 60-66. Cited 50 times.

doi: 10.1016/j.ecolecon.2010.05.001

View at Publisher

$33(2019)$

LCS (2025). Low Carbon Society Blueprint for Iskandar Malaysia 2025. third ed. Access date Dec 12.

http://iskandarmalaysia.com.my/green/download/Low\%20Carbon\%20Society\%20Blueprin t\%20for\%20Iskandar\%20Malaysia\%202025_\%20SPM\%203rd\%20\%20Edition.pdf

34 Leaman, A., Stevenson, F., Bordass, B.

Building evaluation: Practice and principles (Open Access)

(2010) Building Research and Information, 38 (5), pp. 564-577. Cited 160 times. doi: 10.1080/09613218.2010.495217

View at Publisher

35 Lenzen, $M$.

Primary energy and greenhouse gases embodied in Australian final consumption: an input-output analysis

(1998) Energy Policy, 26 (6), pp. 495-506. Cited 351 times. doi: 10.1016/S0301-4215(98)00012-3

View at Publisher

36 Lenzen, M., Peters, G.M.

How City dwellers affect their resource Hinterland

(2010) Journal of Industrial Ecology, 14 (1), pp. 73-90. Cited 131 times. doi: $10.1111 / j .1530-9290.2009 .00190 . x$

View at Publisher

37 Li, G.Z., Niu, S.W., Yang, Z., Zhang, X.

The analysis on environmental and economic cost of rural household energy consumption in loess hilly region of Gansu Province (2008) J. Nat. Resour., 1. Cited 15 times.

38 Long, Y., Yoshida, Y., Dong, L.

Exploring the indirect household carbon emissions by source: Analysis on 49 Japanese cities

(2017) Journal of Cleaner Production, 167, pp. 571-581. Cited 33 times.

https://www.journals.elsevier.com/journal-of-cleaner-production

doi: 10.1016/j.jclepro.2017.08.159

View at Publisher

39 Maji, I.K., Habibullah, M.S., Saari, M.Y.

Financial development and sectoral $\mathrm{CO}_{2}$ emissions in Malaysia

(2017) Environmental Science and Pollution Research, 24 (8), pp. 7160-7176. Cited 30

times.

http://www.springerlink.com/content/0944-1344

doi: 10.1007/s11356-016-8326-1

View at Publisher

40 Majid, M.R., Nordin, A.N., Johar, F., Tifwa, H.Y.

Travel Emission Profile of Iskandar Malaysia Neighbourhoods from Pre1980 s to 2000s (Open Access)

(2014) IOP Conference Series: Earth and Environmental Science, 18 (1), art. no. 012161.

http://www.iop.org/EJ/volume/1755-1315

doi: 10.1088/1755-1315/18/1/012161

View at Publisher 
41 Minx, J.C., Wiedmann, T., Wood, R., Peters, G.P., Lenzen, M., Owen, A., Scott, K., (...), Ackerman, F.

Input-output analysis and carbon footprinting: An overview of applications

(2009) Economic Systems Research, 21 (3), pp. 187-216. Cited 309 times. doi: 10.1080/09535310903541298

View at Publisher

42 Minx, J., Baiocchi, G., Wiedmann, T., Barrett, J., Creutzig, F., Feng, K., Förster, M., (...), Hubacek, K.

Carbon footprints of cities and other human settlements in the UK (Open Access)

(2013) Environmental Research Letters, 8 (3), art. no. 035039. Cited 195 times. http://iopscience.iop.org/1748-9326/8/3/035039/pdf/1748-9326_8_3_035039.pdf doi: $10.1088 / 1748-9326 / 8 / 3 / 035039$

View at Publisher

43 Mulrow, J., Machaj, K., Deanes, J., Derrible, S.

The state of carbon footprint calculators: An evaluation of calculator design and user interaction features

(2019) Sustainable Production and Consumption, 18, pp. 33-40. Cited 19 times. http://www.journals.elsevier.com/sustainable-production-and-consumption/ doi: 10.1016/j.spc.2018.12.001

View at Publisher

44 Muñiz, I., Dominguez, A.

The impact of urban form and spatial structure on per capita carbon footprint in u.S. larger metropolitan areas (Open Access)

(2020) Sustainability (Switzerland), 12 (1), art. no. 389, pp. 1-19. Cited 6 times. https://www.mdpi.com/2071-1050/12/1/389/pdf doi: $10.3390 /$ su 12010389

View at Publisher

45 Ottelin, J., Heinonen, J., Junnila, S.

Carbon footprint trends of metropolitan residents in Finland: How strong mitigation policies affect different urban zones

(2018) Journal of Cleaner Production, 170, pp. 1523-1535. Cited 36 times. doi: 10.1016/j.jclepro.2017.09.204

View at Publisher

46 Phang, F.A., Yoke, W.W., Siong, H.C., Musa, A.N.

Achieving low carbon society through primary school ecolife challenge in Iskandar Malaysia

(2017) Chemical Engineering Transactions, 56, pp. 415-420. Cited 7 times. http://www.aidic.it/cet/17/56/070.pdf

ISBN: 978-889560847-1

doi: $10.3303 /$ CET1756070

View at Publisher

47 Poortinga, W., Pidgeon, N.

Public Perceptions of Risk, Science and Governance: Main Findings of a British Survey of Five Risk Cases

(2003). Cited 106 times.

psych.cf.ac.uk/understandingrisk/docs/survey_2002.pdf UEA/MORI Norwich Retrieved

from

https://sustainabledevelopment.un.org/content/documents/Agenda21.pdf

48 Rizzo, A., Glasson, J.

Iskandar Malaysia

(2012) Cities, 29 (6), pp. 417-427. Cited 44 times.

doi: 10.1016/j.cities.2011.03.003

View at Publisher 
$\square 49$ Saboori, B., Rasoulinezhad, E., Sung, J.

The nexus of oil consumption, $\mathrm{CO}_{2}$ emissions and economic growth in China, Japan and South Korea

(2017) Environmental Science and Pollution Research, 24 (8), pp. 7436-7455. Cited 37

times.

http://www.springerlink.com/content/0944-1344

doi: 10.1007/s11356-017-8428-4

View at Publisher

50 Salo, M., Savolainen, H., Karhinen, S., Nissinen, A.

Drivers of household consumption expenditure and carbon footprints in

Finland (Open Access)

(2021) Journal of Cleaner Production, 289, art. no. 125607. Cited 2 times.

https://www.journals.elsevier.com/journal-of-cleaner-production

doi: $10.1016 /$ j.jclepro.2020.125607

View at Publisher

51 Sarker, M.N.I., Hossin, M.A., Hua, Y.X., Anusara, J., Warunyu, S., Chanthamith, B. Low carbon city development in China in the context of new type of urbanization (2018) Low Carbon Econ., 9 (1), pp. 45-61. Cited 4 times.

52 Shan, S., Bi, X.

Low Carbon Development of China's Yangtze River Delta Region

(2012) Problemy Ekorozwoju, 7 (2), pp. 33-4l. Cited 26 times.

http://ekorozwoj.pol.lublin.pl/nol4/e.pdf

53 Siong, H.C., Matsuoka, Y.

Low Carbon Society Blueprint for Iskandar Malaysia 2025

(2013). Cited 15 times.

UTM-Low Carbon Asia Research Center, Universiti Teknologi Malaysia Johor, Malaysia

54 Song, K., Qu, S., Taiebat, M., Liang, S., Xu, M.

Scale, distribution and variations of global greenhouse gas emissions driven by U.S. households (Open Access)

(2019) Environment International, Part A 133, art. no. 105137. Cited 11 times.

www.elsevier.com/locate/envint

doi: 10.1016/j.envint.2019.105137

View at Publisher

55 Tan, S., Yang, J., Yan, J., Lee, C., Hashim, H., Chen, B.

A holistic low carbon city indicator framework for sustainable development

(2017) Applied Energy, Part 2 185, pp. 1919-1930. Cited 104 times. http://www.elsevier.com/inca/publications/store/4/0/5/8/9/1/index.htt doi: 10.1016/j.apenergy.2016.03.041

View at Publisher

56 Wang, C., Zhan, J., Li, Z., Zhang, F., Zhang, Y.

Structural decomposition analysis of carbon emissions from residential consumption in the Beijing-Tianjin-Hebei region, China

(2019) Journal of Cleaner Production, 208, pp. 1357-1364. Cited 25 times.

https://www.journals.elsevier.com/journal-of-cleaner-production

doi: $10.1016 /$ j.jclepro.2018.09.257

View at Publisher 
57 Wang, L., Zhang, F., Pilot, E., Yu, J., Nie, C., Holdaway, J., Yang, L., (...), Krafft, T.

Taking action on air pollution control in the Beijing-Tianjin-Hebei (BTH) region: Progress, challenges and opportunities (Open Access)

(2018) International Journal of Environmental Research and Public Health, 15 (2), art. no. 306. Cited 57 times.

http://www.mdpi.com/1660-4601/15/2/306/pdf

doi: 10.3390/ijerph15020306

View at Publisher

58 Ward, J.D., Sutton, P.C., Werner, A.D., Costanza, R., Mohr, S.H., Simmons, C.T.

Is decoupling GDP growth from environmental impact possible?

(Open Access)

(2016) PLoS ONE, 11 (10), art. no. 0164733. Cited 156 times.

http://journals.plos.org/plosone/article/asset?id=10.1371/journal.pone.0164733.PDF

doi: $10.1371 /$ journal.pone.0164733

View at Publisher

59 Weber, C.L., Matthews, H.S.

Quantifying the global and distributional aspects of American household carbon footprint

(2008) Ecological Economics, 66 (2-3), pp. 379-391. Cited 358 times.

doi: 10.1016/j.ecolecon.2007.09.021

View at Publisher

60 Weber, C., Perrels, A.

Modelling lifestyle effects on energy demand and related emissions

(2000) Energy Policy, 28 (8), pp. 549-566. Cited 209 times.

doi: $10.1016 / 50301-4215(00) 00040-9$

View at Publisher

61 Wei, Y.-M., Liu, L.-C., Fan, Y., Wu, G.

The impact of lifestyle on energy use and $\mathrm{CO}_{2}$ emission: An empirical analysis of China's residents

(2007) Energy Policy, 35 (1), pp. 247-257. Cited 222 times.

doi: 10.1016/j.enpol.2005.11.020

View at Publisher

62 CO2 Emissions (Metric Tons Per Capita)

(2014). Cited 24 times.

A ccessed August 28

https://data.worldbank.org/indicator/en.atm.co2e.pc

63 Xiao-ling, L., Chun-liang, X., Ping-jun, S.

The research of pattern and mechanism of the chinese urban land use intensity under new type urbanization

(2015) World Reg. Stud., 1, pp. 60-67.

64 Yao, L., Liu, J., Wang, R.

Comparison and analysis of carbon emissions embodied in household consumption

between urban and rural area of China

(2011) Chin. J. Popul. Resour. Environ., 24 (4), pp. 25-29. Cited 6 times.

65 Zahari, M.A.Z., Majid, M.R., Ho, C.S., Kurata, G., Nadhirah, N., Irina, S.Z.

Relationship between land use composition and $\mathrm{PM}_{10}$ concentrations in Iskandar Malaysia

(2016) Clean Technologies and Environmental Policy, 18 (8), pp. 2429-2439. Cited 6 times. https://link.springer.com/journal/10098

doi: $10.1007 / \mathrm{s} 10098-016-1263-3$

View at Publisher 
$\square 66$ Zen, I.S., Al-Amin, A.Q., Doberstein, B.

Mainstreaming climate adaptation and mitigation policy: Towards multi-level climate governance in Melaka, Malaysia (Open Access)

(2019) Urban Climate, 30, art. no. 100501. Cited 9 times.

http://www.journals.elsevier.com/urban-climate/

doi: $10.1016 /$ j.uclim.2019.100501

View at Publisher

67 Zhao, C.-S., Niu, S.-W., Zhang, X.

Effects of household energy consumption on environment and its influence factors in rural and urban areas (Open Access)

(2012) Energy Procedia, 14, pp. 805-811. Cited 39 times.

doi: 10.1016/j.egypro.2011.12.887

View at Publisher

68 Zheng, Y.

Socio-economic development and carbon emissions

(2009) IARU Int. Sci. Congr. Clim. Change

Copenhagen, Denmark 10-12, March 2009

$\frown$ Al-Amin, A.Q.; Department of Geography and Environmental Management, University of Waterloo, ON, Canada; email:qalamin@uwaterloo.ca

(C) Copyright 2021 Elsevier B.V., All rights reserved.

\section{About Scopus}

What is Scopus

Content coverage

Scopus blog

Scopus API

Privacy matters

\section{Language}

日本語に切り替える

切换到简体中文

切換到繁體中文

Русский язык

\section{Customer Service}

Help

Contact us

\section{ELSEVIER}

Terms and conditions $\pi$ Privacy policy $\pi$

Copyright (C) Elsevier B.V ז. All rights reserved. Scopus ${ }^{\circledR}$ is a registered trademark of Elsevier B.V.

We use cookies to help provide and enhance our service and tailor content. By continuing, you agree to the use of cookies. 